Água salina e condicionador de solo em beterraba

D'OlIVEIRA, L. O. B. ; RESENDE, G. M. de; FLORI, J. E. Produtividade do aspargo sob irrigação na região do Submédio São Francisco. Horticultura Brasileira, Brasilia, v. 17, n. 1, p. 41-44, março 1999.

\title{
Produtividade do aspargo sob irrigação na região do Submédio São Francisco.
}

\author{
Lúcio O. B. D’Oliveira; Geraldo M. de Resende; José Egidio Flori \\ Embrapa Semi-Árido, Caixa Postal 23, 56.300-000 Petrolina - PE.
}

\section{RESUMO}

O objetivo do presente trabalho foi avaliar uma coleção de cultivares e híbridos de aspargo (Asparagus officinalis L.), quanto à produtividade e adaptabilidade às condições do Submédio São Francisco. A avaliação foi realizada no Campo Experimental de Bebedouro (Embrapa Semi-Árido), em Petrolina - PE, durante o período de 1991 a 1994. A coleção, constituída por duas cultivares e doze híbridos provenientes de Pelotas - RS, dez híbridos da França e três híbridos dos Estados Unidos, foi implantada em 15 de agosto de 1990. Na avaliação da produtividade, considerou-se apenas os turiões brancos, retos, sem manchas ou lesões, com diâmetro acima de 8 mm e comprimento variando de 15 a $20 \mathrm{~cm}$. A produtividade média por genótipo durante o período de 1991 a 1994 variou de 1,74 a 6,33 t. ha ${ }^{-1}$. O ano de 1992 destacou-se com a maior produtividade média $\left(4,46\right.$ t. ha $\left.{ }^{-1}\right)$. Os híbridos de origem francesa apresentaram uma acentuada mortalidade de plantas, indicando uma provável inadaptação às condições da região. Os híbridos W 12 x 14, G 4 x 14 e G 103 x 14, procedentes de Pelotas, destacaram-se com produtividades médias superiores a 4,40 t. ha- ${ }^{-1}$. A cultivar New Jersey 220 apresentou o melhor desempenho, com 3,69 t. ha ${ }^{-1}$. Estes dados indicam o aspargo como uma boa alternativa agrícola para os perímetros irrigados da região Nordeste do Brasil, oferecendo, inclusive, condições de a região competir nos mercados interno e externo.

Palavras-chave: Asparagus officinalis L., cultivares, híbridos, adaptabilidade.

\begin{abstract}
Asparagus yield under irrigation at the Submédio São Francisco region.

The objectives of this study were to evaluate genotypes of asparagus (Asparagus officinalis L.), in terms of yield and adaptation to conditions of the Submédio São Francisco region. The research was conducted between 1991 and 1994 at the Bebedouro Experimental Station of Embrapa Semi-Árido, in Petrolina, Pernambuco State. Two standard cultivars and twelve Brazilian hybrids (Pelotas and Rio Grande do Sul), ten French hybrids, and three hybrids from the United States were tested. Only straight spears, without stains or lesions, and diameter above eight $\mathrm{mm}$ and length varying from 15 to $20 \mathrm{~cm}$, were considered for determining yield. The mean annual productivity per genotype varied from 1.74 to $6.33 \mathrm{t}$. ha $\mathrm{a}^{-1}$ during the period 1991 to 1994. In 1992 the highest performance was observed, with a productivity of $4.46 \mathrm{t}$. ha ${ }^{-1}$. The hybrids from France presented an accentuated mortality, indicating a probable lack of adaptation to the local conditions. The hybrids W $12 \times 14, \mathrm{G} 4 \times 14$ and G 103 $\mathrm{x} 14$, from Pelotas, yielded above $4.40 \mathrm{t}$. ha ${ }^{-1}$. The variety New Jersey 220 showed the best performance, with $3.69 \mathrm{t}^{\text {. ha }}{ }^{-1}$. These data indicate that asparagus cropping is a good alternative for the irrigation projects in the Brazilian Northeast, offering conditions for competition in internal and external markets.
\end{abstract}

Keywords: Asparagus officinalis L., cultivars, hybrids, adaptability.

(Aceito para publicação em 09 de novembro de 1998) 
$\mathrm{C}$ om a introdução do aspargo (Asparagus officinalis L.), como alternativa de cultura para estabilização do sistema produtivo nos projetos de irrigação do Submédio São Francisco, na década de 1980, tornou-se necessário avaliar diferentes genótipos, objetivando verificar seu comportamento produtivo e o grau de adaptabilidade às condições de clima da região semi-árida do Brasil, hoje com uma área cultivada de 500 hectares, destacando-se como uma das maiores regiões produtoras desta cultura no país.

O rendimento anual de um aspargal em um ano ou no período de vários anos é determinado por uma série de fatores como a idade da coroa, condições climáticas, solo, irrigação, pragas, doenças e manejo da cultura, apresentado em média, um período de 12 a 15 anos, durante o qual os rendimentos são economicamente rentáveis (D’Oliveira, 1992).

No Rio Grande do Sul, tradicional região produtora de aspargo, onde vem sendo cultivado desde a década de 30,0 rendimento médio das lavouras oscila entre 1,50 e 2,00 t. ha-1 (Oliveira et al., 1981). Em levantamentos nas áreas de diversos produtores na região de Pelotas, Oliveira \& Bianchini (1982), verificaram produtividades médias variando de 2,00 a 2,30 t. ha- .

Nas condições de clima semi-árido, no vale do São Francisco, D’Oliveira \& Oliveira (1984), verificaram produtividades variando de 3,80 a 5,10 t. ha- ${ }^{-1}$ para diferentes híbridos e cultivares de aspargo. Em condições semelhantes, Marciani-Bendezú et al. (1995), relatam produtividades acima de 2,30 t. ha-1, destacando-se o híbrido G 10 x 14 com 3,30 t. ha ${ }^{-1}$.

Comparando quinze cultivares de aspargo na Polônia, no espaçamento de $1,60 \times 0,35$ m, Knaflewski (1990), verificou produtividades variando de 2,33 a 9,18 t. ha ${ }^{-1}$, tendo a cultivar Franklin se destacado com a maior produtividade $\left(9,18\right.$ t. ha $\left.{ }^{-1}\right)$ e a UC-72 com a menor $\left(2,33 \mathrm{t} . \mathrm{ha}^{-1}\right)$. Avaliando vinte cultivares de aspargo originárias da Polônia, Holanda, França e USA, Knaflewski \& Konys (1993) verificaram que a cultivar Mary Washington apresentou baixo rendimento, assim como as cultivares UC-72 e UC-800, sendo consideradas inadequadas para o cultivo nas condições da Polônia. As cultivares holandesas e alemãs mostraram-se mais produtivas que as francesas, à exceção do primeiro ano de colheita. Resultados similares foram obtidos por Knaflewski (1996) com relação a cultivar UC-157 que tambem apresentou baixa produtividade, juntamente com as cultivares Larac, Tainan n. ${ }^{\circ} 1$ e Del Monte 361. Na Alemanha, Paschold et al. (1996), comparando doze cultivares de aspargo da Alemanha, Holanda, França, Espanha, USA e Taiwan, observaram produtividades variando de 0,47 a 4,20 t. ha ${ }^{-1}$, para o segundo ano e 0,18 a 6,16 t. ha ${ }^{-1}$, tendo a cultivar holandesa Gijnlim atingido o maior rendimento e a cultivar UC-157 apresentado o pior desempenho.

No Peru, Delgado de la Flor et al. (1996), relatam rendimentos de até 22,0 t.ha ${ }^{-1}$ com uma produtividade média de $5,6 \mathrm{t}$. ha- ${ }^{-1}$ para as cultivares Mary Washington e UC-157, que representam, respectivamente, 55 e $45 \%$ da atual área plantada com aspargo.

Na Itália, Gasperetti (1996), relata produtividade de $13,1 \mathrm{t}$. ha-1 para a cultivar Grande, colocando-a como uma nova alternativa de plantio a cultivar UC-157 que mostrou-se menos produtiva. Ao contrario, Branca (1997) testando treze cultivares na Sicília - Itália, verificou uma produtividade média de 5,3 t. ha- ${ }^{-1}$, sendo que os rendimentos mais altos foram obtidos pelas cultivares UC-157 (12,0 t. ha $^{-1}$ ) e JM2002 (7,5 t. ha-1)

Objetivou-se neste trabalho avaliar uma coleção de cultivares e híbridos de aspargo, quanto à produtividade e adaptabilidade às condições do Submédio São Francisco.

\section{MATERIAL E MÉTODOS}

O trabalho foi instalado no Campo Experimental de Bebedouro, em Petrolina - $\mathrm{PE}$ à altitude de $365,5 \mathrm{~m}$ e temperatura média anual de $26^{\circ} \mathrm{C} \mathrm{em} \mathrm{um}$ Latossolo vermelho-amarelo, textura franco arenosa. A coleção avaliada, constituída por duas cultivares (New Jersey 220 e Waltham Washington), doze híbridos provenientes da Embrapa Clima Temperado, Pelotas - RS (G 4 x 14, G $10 \times 14$, G $19 \times 14, \mathrm{G} 21 \times 14, \mathrm{G}$
$22 \times 14, \mathrm{G} 23 \times 14, \mathrm{G} 27 \times 14, \mathrm{G} 101 \times$ 14, G $102 \times 14$, G $103 \times 14$, W 7 × 14 e W 12 x 14) e dez híbridos provenientes da França (Junon, Minerve, Larac, Mira, Diane, Aneto, Desto, Cito, Bruneto e Staline) foi inicialmente implantada em 15 de agosto de 1990. Um ano após foram introduzidos três híbridos provenientes dos Estados Unidos (UC-72, UC711 e UC-157-F 2 ). A adubação incorporada ao solo, constou de $10 \mathrm{t}$. ha $\mathrm{ha}^{-1} \mathrm{de}$ esterco de caprino juntamente com 400 $\mathrm{kg} / \mathrm{ha}$ de superfosfato triplo no plantio. Um mês após, foram aplicados $220 \mathrm{~kg}$ / ha de uréia e $490 \mathrm{~kg} / \mathrm{ha}$ de cloreto de potássio. Seis meses depois, foram repetidas as aplicações de uréia e cloreto de potássio. As adubações de manutenção e produção foram na proporção de 100,50 e $220 \mathrm{~kg} /$ ha de $\mathrm{N}, \mathrm{P}_{2} \mathrm{O}_{5}$ e $\mathrm{K}_{2} \mathrm{O}$, respectivamente, a cada seis meses.

O espaçamento utilizado foi de 2,30 $\mathrm{m}$ entre fileiras e $0,40 \mathrm{~m}$ entre plantas, com 50 plantas por parcela, não sendo utilizado qualquer delineamento experimental.

As irrigações foram realizadas por aspersão convencional, com aplicação média de $15 \mathrm{~mm}$ de água duas vezes por semana.

A cultura foi mantida no limpo através da aplicação do herbicida Diuron e capinas manuais, não sendo realizados quaisquer tratos fitossanitários contra pragas ou doenças.

A colheita foi realizada doze meses após o transplantio, iniciando-se cinco dias após o corte da parte aérea, sendo no primeiro ano (1991 e 1992 respectivamente para os primeiros genótipos implantados e genótipos procedentes dos Estados Unidos), realizada por um período de dez dias e nos anos subsequentes, por um período de 20 dias.

$\mathrm{Na}$ avaliação da produtividade, considerou-se apenas os turiões brancos, retos, com pontas perfeitas, escamas fechadas e aderentes, sem manchas ou lesões e com diâmetro acima de $8 \mathrm{~mm}$, com comprimento variando de 15 a $20 \mathrm{~cm}$.

\section{RESULTADOS E DISCUSSÃO}

A produtividade média dos genótipos durante o período de 1991 a 1994, variou de 1,74 a 6,33 t. ha ${ }^{-1}$, tendo o ano de 1992 alcançado a maior pro- 
Tabela 1. Produtividade de turiões de cultivares e híbridos de aspargos. Petrolina, Embrapa Semi-Árido, 1991/94

\begin{tabular}{|c|c|c|c|c|c|c|}
\hline \multirow{2}{*}{ Genótipos } & \multirow{2}{*}{ Procedência } & 1991 & 1992 & 1993 & 1994 & Média \\
\hline & & \multicolumn{5}{|c|}{ t.ha ${ }^{-1}$} \\
\hline New Jersey-220 & Brasil & 1,80 & 4,81 & 5,37 & 2,78 & 3,69 \\
\hline W. Washington & Brasil & 2,61 & 4,48 & 3,92 & 1,52 & 3,13 \\
\hline Média & & 2,21 & 4,65 & 4,65 & 2,15 & 3,41 \\
\hline G $4 \times 14$ & Brasil & 3,17 & 6,17 & 5,99 & 3,16 & 4,62 \\
\hline G $10 \times 14$ & Brasil & 2,20 & 4,56 & 5,30 & 2,00 & 3,52 \\
\hline G $19 \times 14$ & Brasil & 1,99 & 4,51 & 4,08 & 2,44 & 3,25 \\
\hline G $21 \times 14$ & Brasil & 1,69 & 4,60 & 4,55 & 2,14 & 3,25 \\
\hline G $22 \times 14$ & Brasil & 1,81 & 4,52 & 4,49 & 3,23 & 3,51 \\
\hline G $23 \times 14$ & Brasil & 2,42 & 3,26 & 3,09 & 1,34 & 2,53 \\
\hline G $27 \times 14$ & Brasil & 2,69 & 6,81 & 3,34 & 1,50 & 3,58 \\
\hline G $101 \times 14$ & Brasil & 2,43 & 5,47 & 5,22 & 1,73 & 3,71 \\
\hline G $102 \times 14$ & Brasil & 2,95 & 5,20 & 2,50 & 0,86 & 2,88 \\
\hline G $103 \times 14$ & Brasil & 3,93 & 6,68 & 4,92 & 2,10 & 4,41 \\
\hline W 7 x 14 & Brasil & 1,80 & 5,42 & 2,99 & 1,16 & 2,84 \\
\hline W $12 \times 14$ & Brasil & 1,95 & 6,97 & 7,59 & 3,70 & 5,05 \\
\hline Média & & 2,42 & 5,35 & 4,51 & 2,11 & 3,60 \\
\hline Diane & França & 2,34 & 3,24 & 0,59 & - & 2,06 \\
\hline Junon & França & 2,00 & 2,72 & 2,34 & - & 2,35 \\
\hline Minerve & França & 2,25 & 3,46 & 1,61 & - & 2,44 \\
\hline Larac & França & 3,46 & 3,30 & 2,14 & - & 2,97 \\
\hline Mira & França & 2,81 & 3,82 & 5,30 & - & 3,98 \\
\hline Aneto & França & 5,61 & 7,34 & 6,04 & - & 6,33 \\
\hline Cito & França & 4,05 & 4,37 & 3,16 & - & 3,86 \\
\hline Desto & França & 3,82 & 5,63 & 3,94 & - & 4,46 \\
\hline Bruneto & França & 5,41 & 5,07 & 6,24 & - & 5,58 \\
\hline Steline & França & 4,67 & 4,23 & 5,23 & - & 4,71 \\
\hline Média & & 3,64 & 4,32 & 3,66 & - & 3,87 \\
\hline UC - 72 & EUA & - & 1,25 & 1,53 & 3,01 & 1,93 \\
\hline UC - 711 & EUA & - & 0,79 & 1,51 & 2,92 & 1,74 \\
\hline $\mathrm{UC}-157-\mathrm{F}_{2}$ & EUA & - & 1,64 & 1,43 & 3,19 & 2,08 \\
\hline Média & & - & 1,23 & 1,49 & 3,04 & 1,92 \\
\hline Média Geral & & 2,91 & 4,46 & 3,87 & 2,28 & 3,50 \\
\hline
\end{tabular}

dutividade média $(4,46 \mathrm{t}$. ha-1) (Tabela 1). Os híbridos de origem francesa, apesar de produtivos, apresentaram uma acentuada e progressiva mortalidade de plantas com conseqüente queda da produtividade na maioria dos materiais em 1993 (Tabela 1), indicando uma provável inadaptação às condições do Submédio São Francisco. Portanto não são recomendados para o plantio, mesmo por um curto período de três anos devido ao alto custo de implantacão da cultura, razão pela qual em 1994 deixa- ram de ser avaliados. Entretanto, os híbridos de procedência nacional W $12 \mathrm{x}$ 14, G 4 × 14 e G 103 x 14 destacaramse com produtividades médias nos anos em estudo superiores a 4,4 t. ha-1, demonstrando uma boa adaptação às condições do Submédio São Francisco, confirmando as observações de Silveira \& Augustin (1993), da maior produtividade apresentada pelos híbridos. Entre as cultivares, a New Jersey 220 apresentou o melhor desempenho, com 3,69 t. ha $^{-1}$ comparada a Waltham Washington com 3,13 t. ha-1, todavia, inferior aos híbridos mais produtivos. Estas produtividades são superiores às da cultura obtidas no Sul do Brasil (Oliveira \& Bianchini, 1982; Oliveira et al., 1981), assim como em região semelhante (Marciani-Bendezu et al., 1995). Contudo, são similares aos rendimentos relatados por D'Oliveira \& Oliveira (1984), que verificaram produtividades variando de 3,80 a 5,10 t. ha ${ }^{-1}$ e discordantes das afirmações de Augustin et al. (1990), que verificaram para o híbrido 
G $27 \times 14$ produtividade $40 \%$ superior à da cultivar New Jersey 220 .

Os híbridos de origem dos Estados Unidos apresentaram baixa produtividade em todos os anos, concordando com Knaflewski (1990), Knaflewski \& Konys (1993) e Knaflewski (1996), que também verificaram baixos rendimentos para as cultivares americanas UC72 e UC-157 e discordantes de Branca (1997), que verificou altas produtividades para a cultivar UC-157.

Produtividades bem superiores às obtidas no presente trabalho têm sido relatadas por Delgado de La Flor \& Van Oordt (1996), Gasperetti (1996) e Branca (1997), para diferentes cultivares em diversas partes do mundo; contudo, usando maiores densidades populacionais. Deve-se salientar que, conforme relatam Oliveira et al. (1970) e Moran \& Issacs (1960), o aumento da densidade promove uma maior produtividade. No presente trabalho, a densidade populacional de 10.869 plantas/ha, está muito abaixo das populações recomendadas por Williams \& Kendall (1976), que são de 25.000 a 37.000 plantas/ha e inferior às de Tanaka \& Genta (1983) de 18.000 plantas/ha, assim como as sugeridas por Toledo (1990) de 15.552 a 16.667 plantas/ha. Salienta-se ainda que Toledo (1990), encontrou produtividade de 8,00 t. ha-1/ano, realizando colheitas a cada seis meses. Baseado nestes dados, há uma grande possibilidade de se incrementar as produtividades obtidas no presente trabalho estudando-se diferentes populações de plantas, consolidando definitivamente o aspargo como uma boa alternativa de exploração agrícola nos perímetros irrigados da região Nordeste do Brasil, oferecendo inclusive, condições da região competir nos mercados interno e externo.

\section{LITERATURA CITADA}

AUGUSTIN, E.; MORAES, E.C.; OSORIO, V.A.; COUTO, M.E.O.; PETERS, J.A.; SALLES, L.A.B. A cultura do aspargo. Pelotas: EMBRAPA-CNPFT, 1990. 24 (Circular Técnica 15).

BRANCA, F. Confronto tra genotipi di asparago in Sicilia. Informatore Agrario, v. 53, n. 39, p. $43-45,1997$.

DELGADO DE LAFLOR, F; VAN OORDT, E. Asparagus in Peru. Acta Horticulturae, n. 415, p. 81 - 85, 1996

D'OLIVEIRA, L.O.B. A cultura do aspargo irrigado na região do Submédio São Francisco. Petrolina: EMBRAPA-CPATSA, 1992 22 p. (Circular Tecnica 26)

D'OLIVEIRA, L.O.B.; OLIVEIRA, J.J. Competição de dez híbridos e uma cultivar de aspargo (Asparagus officinalis L.) no Vale do São Francisco. In: CONGRESSO BRASILEIRO DE OLERICULTURA, 24, 1984, Jaboticabal. Resumos e Palestras.. Jaboticabal: SOB, 1984. p.19

GASPERETTI, L. Confronto tra tre ibridi in coltivazione nel sud, centro e nord Itália. Informatore Agrario, v. 52, n. 39, p. 59 - 62, 1996

KNAFLEWSKI, M. Comparison of the suitability of asparagus cultivars for blanched and green spear production. Acta Horticulturae, n. 271, p. $191-196,1990$.

KNAFLEWSKI, M. Results of the internacional asparagus cultivar trial in Poland. Acta Horticulturae, n. 415, p. 393 - 398, 1996.

KNAFLEWSKI, M.; KONYS, E. Suitability of asparagus culitvars for blanched spear production in a temperate climate. Folia Horticulturae, v. 5, n. 2, p. 23 - 32, 1993.

MARCIANI-BENDEZU, J.; RESENDE, G.M. de; OLIVEIRA, J.J. Avaliação preliminar da cultura do aspargo no norte de Minas Gerais. Horticultura Brasileira, Brasilia, v. 13, n. 2, p. $206-208,1995$

MORAN, C.H.; ISSACS, R.L. Effect of crown spacing on the yield of asparagus. Proceedings of the American Society for Horticultural Science, v. 75, p. 416 - 418, 1960

OLIVEIRA, E. A.; BIANCHINI, C. Diagnóstico e recomendações para a cultura do aspargo na zona produtora da região Sudeste do Rio Grande do Sul. Pelotas: EMBRAPA-UEPAE de Pelotas, 1982. 43 p. (EMBRAPA-UEPAE de Pelotas. Documentos, 13).
OLIVEIRA, E.A.; OLIVEIRA, J.J.; MORAES, E.E.; MAGNANIM, M.; FEHN, L. M.; FELICIANO, A. A cultura do aspargo. Pelotas: EMBRAPA - UEPAE de Cascata, 1981. 48 p. (Circular Téenica 5).

OLIVEIRA, J.J.; OLIVEIRA, E.A.; COSTA, D.M.; GARCIA, A.; SANTOS, A.M.; SILVA, A.R.D. Influência do espaçamento sobre o rendimento de turiões de aspargo. Pelotas: IPEAS, 1970. 3 p. (Indicação e Pesquisa 68).

PASCHOLD, P.J.; HERMMANN, G.; ARTELT, B. Comparison of internacional asparagus cultivars under Rhine-Valley conditions in Germany. Acta Horticulturae, n. 415, p. 257 - 262, 1996.

SILVEIRA, R.F.; AUGUSTIN, E. Relações entre diâmetro e número de hastes, altura e vigor de plantas e produtividade de aspargo. Pesquisa Agropecuária Brasileira, Brasília, v. 28, n. 1, p. 129 - 133, 1993.

TANAKA, M.; GENTA, H. Técnicas de cultivo del esparrago: Report of Japanese Experts for the Japan-Uruguay Vegetable Research Cooperation Project. [S.L.]: Japan International Cooperation Agency, 1983, p. $103-118$.

TOLEDO, J. Asparagus production in Peru. Acta Horticulturae, n. 271, p. 203 - 210, 1990.

WILLIAMS, J.B.; KENDALL, M. Cultural studies of asparagus with reference to beds, ridging and spacing. Experimental Horticulture, v. 28, n. 1, p. 1 - 14, 1976. 